\title{
EFEITO DA RADIAÇÃO GAMA NA ABSORÇÃO DE ÁGUA E NO TEMPO DE COCÇÃO EM CULTIVARES DE SOJA $\left({ }^{(\mathbf{1}}\right)$
}

\author{
TAÍS CAROLINA FRANQUEIRA DE TOLEDO $\left({ }^{2}\right)$; SOLANGE GUIDOLIN CANNIATTI-BRAZACA $\left({ }^{3 *}\right)$; \\ VALTER ARTHUR $\left({ }^{4}\right)$; SONIA MARIA DE STEFANO PIEDADE $\left({ }^{5}\right)$
}

\begin{abstract}
RESUMO
A soja é uma das mais importantes oleaginosas cultivadas no mundo. Seu alto teor de proteínas a torna alternativa importante para a alimentação animal e humana, além de seus subprodutos. Embora no Brasil haja grande produção de soja, ocorrem muitas perdas devido a alterações nas características físico-químicas, resultando em produto com elevada resistência ao cozimento. Vários métodos podem ser empregados para minimizar esse efeito, dentre os quais a radiação ionizante com Cobalto-60 que constitui um método seguro e eficaz para o aumento no tempo de vida útil dos alimentos. Devido a importância comercial da soja, possíveis alterações promovidas pelo tratamento de irradiação devem ser estudadas. O presente trabalho visou à avaliação das alterações na capacidade de hidratação e no tempo de cocção das variedades de soja BRS 212, BRS 213, BRS 214, BRS 231 e E48 submetidas a doses de 2, 4 e 8 kGy de radiação gama. A quantidade de água absorvida pelos grãos variou de 14,00 a 16,66 mL, e o tempo de cocção de 119,67 a 291,33 minutos. A irradiação causou aumento da velocidade de hidratação, mas não da quantidade de água absorvida. O tempo de cocção diminuiu com o aumento da dose irradiada.
\end{abstract}

Palavras-chave: soja, radiação gama, tempo de cocção, hidratação.

\section{ABSTRACT \\ EFFECTS OF GAMMA RADIATION IN THE WATER ABSORPTION AND THE COOKING TIME ON THE SOYBEAN CULTIVARS}

\begin{abstract}
The soybean is one of the most important legume cultivated in the world. Its high protein amount is attractive in the animal feeding and human, beyond its by-products. Although Brazil has great production of soybean, have many losses due the occurrence of alterations in the physico-chemical characteristics, resulting in product that has high resistance to cooking. Some methods can be used to minimize this effect, and the ionizing radiation with Cobalto-60 constitutes a safe and efficient method for the increase in the time of useful life of foods. Due to commercial importance of the soybean, possible alterations promoted for treatment of irradiation must be studied. The present work aimed at the evaluation of the
\end{abstract}

( $\left.{ }^{1}\right)$ Recebido para publicação em 26 de maio de 2006 e aceito em 4 de junho de 2007.

$\left({ }^{2}\right)$ Aluna do curso de Mestrado em Ciência e Tecnologia de Alimentos, Departamento de Agroindústria, Alimentos e Nutrição, Escola Superior de Agricultura "Luiz de Queiroz", Universidade de São Paulo, ESALQ/USP, Caixa Postal 9, 13418-900 Piracicaba (SP).

$\left({ }^{3}\right)$ Departamento de Agroindústria, Alimentos e Nutrição, ESALQ/USP, Piracicaba (SP). E-mail: sgcbraza@esalq.usp.br (*) Autora correspondente.

$\left({ }^{4}\right)$ Centro de energia Nuclear na Agricultura, Universidade de São Paulo, Caixa Postal 96, 13400-900 Piracicaba (SP). E-mail: arthur@cena.usp.br

$\left({ }^{5}\right)$ Departamento de Ciências Exatas, ESALQ/USP, Piracicaba (SP). E-mail: piedade@esalq.usp.br 
alterations in the capacity of hidratation and the time of cooking of the varieties of soybean BRS 212, BRS 213, BRS 214, submitted 231 BRS and E48 to the doses of 2, 4 and $8 \mathrm{kGy}$ of radiation gamma. The amount of water absorbed for the grains varied of 14.00 and $16.66 \mathrm{~mL}$, and the time of cooking of 119.67 and 291.33 minutes. The irradiation caused increase of the hidratation speed, but not on the amount of absorbed water. The cooking time decreased with the increased of the irradiated dose.

Key words: soybean, gamma radiation, cooking time, hidratation.

\section{Introdução}

Os custos estimados da irradiação comercial, como tratamento visando a um aumento de vida útil através da desinfestação, são competitivos com outros métodos de fumigação, físicos e térmicos (Dogbevi et al., 2000; NAscimento, 1992; Villavicencio, 1998).

Rios et al. (2003) relatam que durante armazenamento de grãos de feijão, ocorre perda de qualidade com desenvolvimento de casca dura (hard shell) e alteração na cocção (hard-to-cook).

A cultivar, o tempo após a colheita, as condições ambientais, o sistema e a temperatura de armazenamento influenciam no tempo de cocção das leguminosas (CHIARAdia e GOMES, 1997; LAM-SANCHEZ et al., 1990; Lemos et al., 2004; Vieira et al., 1989). Cunha et al. (1993) constataram que a irradiação promove diminuição da dureza das sementes e, também, no tempo de cocção.

O objetivo deste estudo foi avaliar as alterações no tempo de cozimento e hidratação de grãos das cultivares de soja BRS 212, BRS 213, BRS 214, BRS 231 e Embrapa 48 causadas pela irradiação com raios gama nas doses 0, 2, 4 e 8 kGy.

\section{Material e Métodos}

\section{Material}

As cultivares utilizadas são produtos da EMBRAPA Soja - Londrina, safra agrícola de 2003/ 2004 (colheita em março e abril 2003), umidade em torno de $8 \%$ e armazenadas à temperatura ambiente até a irradiação dos grãos (cerca de dois anos após a colheita), mantendo as mesmas condições para cultivares, BRS 212, BRS 213, BRS 214, BRS 231 e E48. As cultivares precoces foram colhidas até 115 dias e as semiprecoces entre 116 e 125 dias após o plantio (Tabela 1).

\section{Irradiação dos grãos}

Os grãos crus foram tratados com raios gama, nas dosagens de 2, 4 e $8 \mathrm{kGy}$, originados de irradiador de Cobalto ${ }^{60}$ (Irradiador Comercial Multipropósito, tipo compacto, com taxa de dose de $12 \mathrm{kGy} /$ hora). A dose efetivamente recebida pelo material foi de 2,09 a 3,02 nas amostras irradiadas com 2 kGy, de 3,50 a 5,08 para as irradiadas com $4 \mathrm{kGy}$ e de 7,04 a 9,12 nas irradiadas com $8 \mathrm{kGy}$, medidas com o dosímetro Amber, Batch: P., espectrofotômetro Genesys-20, com leitura a $603 \mathrm{~nm}$ e temperatura $35^{\circ} \mathrm{C}$. Considerou-se como controle a amostra não irradiada. As amostras foram acondicionadas em sacos de polietileno sob refrigeração a $4{ }^{\circ} \mathrm{C}$ até realização das análises, que foram feitas em triplicata, seis meses após a irradiação dos grãos.

\section{Análises físicas}

\section{Tempo de hidratação}

O tempo de hidratação dos grãos foi determinado à temperatura ambiente com 10 gramas de grãos para $50 \mathrm{~mL}$ de água destilada, com intervalos de medidas de 1 em 1 hora, até que a leitura se mantivesse estável por três vezes consecutivas (MORRIS et al., 1950).

Tabela 1. Características das cultivares avaliadas

\begin{tabular}{|c|c|c|c|c|}
\hline Cultivar & Maturação & Semeadura & Massa de 100 sementes & Umidade \\
\hline & & & $\mathrm{g}$ & $\%$ \\
\hline BRS 212 & precoce & out/nov & 17,8 & $7,96 \pm 0,1$ \\
\hline BRS 213 & precoce & nov/dez & 16,5 & $7,12 \pm 0,2$ \\
\hline BRS 214 & semiprecoce & nov/dez & 15,3 & $8,58 \pm 0,0$ \\
\hline BRS 231 & semiprecoce & out/nov & 15,6 & $9,18 \pm 0,1$ \\
\hline EMBRAPA 48 & semiprecoce & out/nov & 15,0 & $8,38 \pm 0,7$ \\
\hline
\end{tabular}




\section{Tempo de cocção}

Os grãos íntegros foram escolhidos aleatoriamente entre os que foram previamente utilizados para análise do tempo de hidratação. $O$ tempo de cocção foi medido com uso do equipamento de cocção de Mattson, produzido em aço inox, composto por 25 hastes de 82 gramas cada, conforme o proposto por JACKSON e MARSTON (1981). No momento em que o aparelho foi submerso em Becker de 4 litros contendo 2 litros de água destilada fervente iniciouse a contagem do tempo. Para a elaboração dos gráficos acompanhou-se a queda das hastes até que as 25 perfurassem os grãos. A numeração das hastes foi feita de acordo com a ordem em que transpassaram os grãos. O tempo ótimo de cocção foi definido como o tempo necessário para que 13 hastes $(50 \%+1$ haste $)$ perfurassem os grãos.

\section{Análise estatística}

O delineamento experimental empregado foi inteiramente ao acaso, com três repetições por tratamento. Os resultados foram submetidos à análise de variância pelo teste $\mathrm{F}$, e a comparação das médias obtidas nos diferentes tratamentos analisada segundo teste de Tukey $(p<0,05)$. Foram analisados separadamente os efeitos dos diferentes tratamentos utilizados. Para os resultados referentes às quatro doses de irradiação foram utilizadas as médias das cinco cultivares estudadas. Para os resultados referentes às cultivares, foram utilizadas as médias das quatro doses de irradiação, e para o tratamento de cocção utilizou-se a média das quatro doses de irradiação e das cinco cultivares.

\section{Resultados e Discussão}

\section{Tempo de hidratação}

Nas figuras 1 e 2, observam-se as curvas de hidratação dos grãos. Na figura 1, verifica-se que durante as duas primeiras horas a cultivar E 48 foi a que mais absorveu água. A quantidade de água absorvida foi igual à massa inicial dos grãos após duas horas. As cultivares 212 e 231 atingiram o dobro de sua massa com três horas de hidratação, e as cultivares 213 e 214, com quatro horas. Após sete horas de hidratação, a cultivar 212 foi a que acusou a maior absorção de água. Os grãos que atingiram o dobro de sua massa em menos tempo foram também as que absorveram mais água no fim de 8 horas de estudo.

Na primeira hora de hidratação (Figura 2), o tratamento com $8 \mathrm{kGy}$ promoveu maior absorção. Na segunda hora não houve diferença entre nenhuma das doses utilizadas. A partir da terceira hora não houve relação entre dose de radiação e absorção de água.

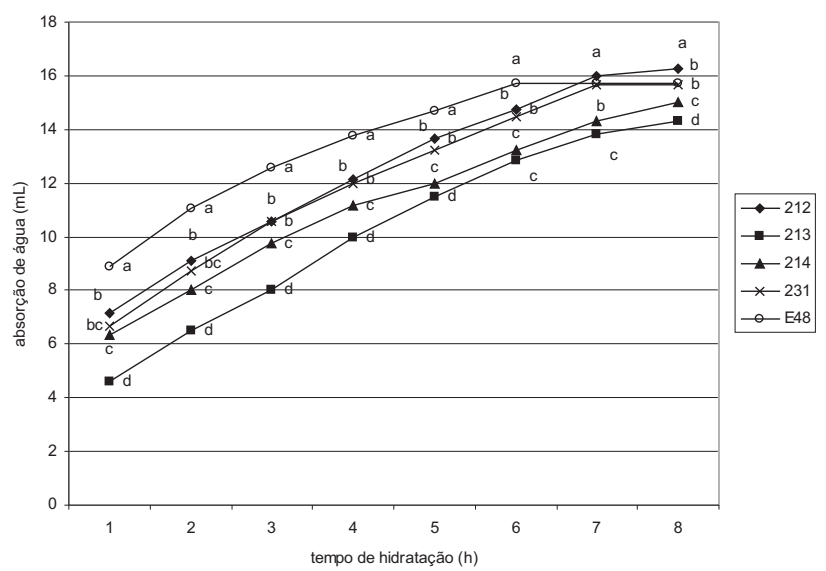

Figura 1. Quantidade de água absorvida no período de tempo de hidratação das cinco cultivares analisadas no experimento $(212,213,214,231$ e E48), obtida através de média das quatro doses analisadas.

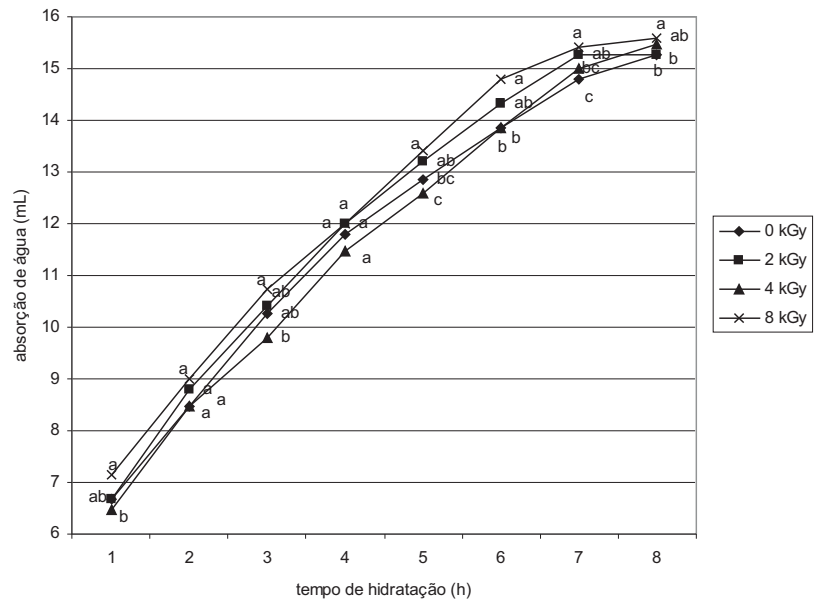

Figura 2. Quantidade de água absorvida no período de tempo de hidratação das quatro doses analisadas no experimento $(0,2,4$ e $8 \mathrm{kGy})$, obtida através de média das cinco cultivares analisadas.

O tempo total para a estabilização da absorção variou entre cultivares e doses (Tabela 2), tendo sido de 8 horas o tempo mínimo verificado para todas as amostras analisadas. A irradiação promoveu diminuição do tempo de hidratação de todas as cultivares. Já em relação à quantidade de água absorvida, as diferenças não tiveram relação com a irradiação. BYUN et al. (1993) verificaram redução do tempo de hidratação em grãos de soja irradiados com doses de até 10 kGy. RAO e VAKIL (1985) atribuíram essa redução à quebra de moléculas de proteínas e carboidratos em moléculas de baixo peso molecular, resultando em aumento da velocidade de absorção. 
Tabela 2. Tempo de hidratação e volume de água destilada absorvida pelos grãos

\begin{tabular}{|c|c|c|c|c|c|c|c|c|}
\hline \multirow{2}{*}{ Cultivares } & \multicolumn{2}{|c|}{ Controle } & \multicolumn{2}{|c|}{ Dose 2 kGy } & \multicolumn{2}{|c|}{ Dose 4 kGy } & \multicolumn{2}{|c|}{ Dose $8 \mathrm{kGy}$} \\
\hline & Tempo & Volume & Tempo & Volume & Tempo & Volume & Tempo & Volume \\
\hline 212 & 10 & $16,3 \pm 0,6$ & 9 & $16,0 \pm 0,0$ & 9 & $16,7 \pm 0,6$ & 9 & $16,0 \pm 0,0$ \\
\hline 213 & 12 & $17,0 \pm 0,0$ & 10 & $14,0 \pm 0,0$ & 10 & $14,0 \pm 0,0$ & 10 & $14,3 \pm 0,6$ \\
\hline 214 & 11 & $16,0 \pm 0,0$ & 11 & $16,0 \pm 0,0$ & 10 & $16,0 \pm 0,0$ & 9 & $14,0 \pm 0,0$ \\
\hline 231 & 9 & $15,0 \pm 0,0$ & 9 & $15,3 \pm 0,0$ & 9 & $15,7 \pm 0,6$ & 8 & $16,3 \pm 0,6$ \\
\hline E48 & 8 & $15,0 \pm 0,6$ & 8 & $16,0 \pm 0,0$ & 8 & $15,0 \pm 0,0$ & 8 & $17,0 \pm 0,0$ \\
\hline
\end{tabular}

De acordo com WANG et al. (1979), o tamanho do grão e a cultivar de soja afetam a velocidade de absorção, sugerindo que cultivares com maiores volumes têm velocidade mais rápida, embora o tipo influencie. Tal afirmação difere do observado no presente trabalho, pois classificando por tamanho temse a seguinte seqüência de cultivares: 212, 213, 231,214 e E48 (do maior para o menor); classificando por velocidade de absorção de água a seqüência foi: E48, 231, 212, 214 e 213 (do mais rápido para o menos rápido). Segundo Esteves et al. (2002), as características do tegumento, como espessura, peso, aderência aos cotilédones, elasticidade, porosidade e propriedades coloidais, interferem na absorção de água. Pode-se afirmar também que existe variação do tempo de máxima hidratação em vista do genótipo e das condições ambientais a que os grãos foram submetidos durante desenvolvimento (CARBONELL et al., 2003).

\section{Tempo de cocção}

Nas figuras de 3 e 4, são apresentados os comportamentos da cocção.

Os grãos da cultivar 212 foram os de cocção mais rápida. As cultivares 214 e 213 foram de cocção mais lenta e as cultivares 231 e E48 tiveram tempo de cocção intermediário. É possível notar relação inversa entre tempo de hidratação e cocção considerando-se as figuras 1 e 3). Esses resultados concordam com os de Rodrigues et al. (2005) que colocam que genótipos que apresentem maior capacidade de absorção de água terão maior facilidade de cozimento. GARCIA-VELA e STANLEY (1989) relatam também que a facilidade de cocção está relacionada com o volume de água absorvido antes do cozimento. A cultivar E48 foi a que absorveu água mais rapidamente (Tabela 2) e com tempo intermediário para cocção, indicando não ser este o único fator determinante.

VIEIRA et al. (1997) observaram tempos de cozimento entre 155 e 219 minutos para soja, sendo inversamente correlacionado com o tempo de absorção de água, ou seja, quanto maior a absorção, menor foi o tempo de cozimento, concordando com os dados das figuras 1 e 3 .

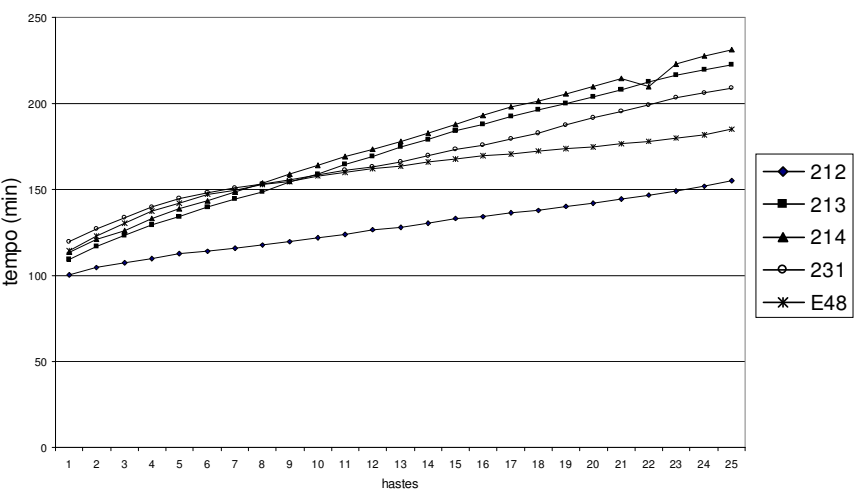

Figura 3.Tempo de cocção das cultivares 212, 213, 214, 231 e E48 (obtido através de média das quatro doses utilizadas).

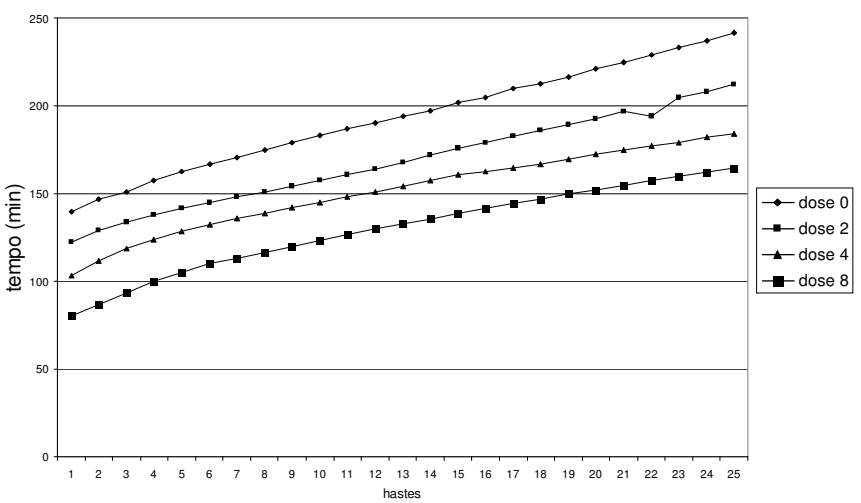

Figura 4. Tempo de cocção das quatro doses analisadas $(0,2,4$ e 8 kGy), obtido através de média das cinco cultivares analisadas.

Segundo Carbonell et al. (2003), que estudaram genótipos de feijão, as diferenças no tempo de cocção se devem a componentes genéticos e ambientais, épocas e locais de cultivo e possivelmente interação destes efeitos. Nos dados constatados, a 
única variação se deve aos genótipos, já que o local e época de plantio são os mesmos. Para DALLa Corte et al. (2003), as qualidades tecnológicas e nutricionais das leguminosas são determinadas pelo genótipo e pelas condições do ambiente durante o desenvolvimento da planta e dos grãos. Portanto, a qualidade para o cozimento é afetada por fatores climáticos com a alta temperatura no período de aumento dos grãos, práticas de cultivo, beneficiamento pós-colheita, condições de armazenamento e tecnologia de processamento.

O tempo médio (haste 13) e o total (25 hastes) de cocção mostraram que a irradiação diminuiu os tempos de cocção conforme aumento das doses.

Novamente nota-se a relação inversa entre tempo de hidratação e cocção dos grãos, visto que as doses que promoveram maior absorção de água tiveram menor tempo de cocção.

IYER et al. (1980) observaram redução de cerca de $50 \%$ no tempo de cocção de feijões macerados e irradiados com dose de $5 \mathrm{kGy}$. No presente trabalho, houve redução de 12,27 \% nas amostras de $2 \mathrm{kGy}$; $23,82 \%$ para as de $4 \mathrm{kGy}$ e $31,96 \%$ para as de $8 \mathrm{kGy}$ em relação aos grãos sem irradiação.

De acordo com os resultados, conclui-se que a irradiação de grãos de soja com raios gama entre 2 e 8 kGy promoveu redução no tempo de hidratação, mas não na quantidade de água absorvida; na média para as cinco cultivares estudadas o aumento da radiação implicou diminuição do tempo de cocção.

\section{Agradecimentos}

À EMBRAPA (Empresa Brasileira de Pesquisa Agropecuária) pela doação dos grãos; ao IPEN (Instituto de Pesquisas Energéticas e Nucleares) pela irradiação; à CAPES (Coordenação de Aperfeiçoamento de Pessoal de Nível Superior) pela concessão de bolsa de estudos.

\section{Referências}

BYUN, M.W.; KWON, J.H.; MORI, T. Improvement of physical properties of soybeans by gama irradiation. Radiation Physics and Chemistry, Oxford, v. 42, n. 1, p. 313-317, 1993.

CHIARADIA, A.C.N.; GOMES, J.C. Feijão: química, nutrição e tecnologia. Viçosa: UFV, 1997. 180p.

CARBONELL, S.A.M.; CARVALHO, C.R.L; PEREIRA, V.R. Qualidade tecnológica de grãos de genótipos de feijoeiro cultivados em diferentes ambientes. Bragantia, Campinas, v.62, n. 3, p. $369-379,2003$.
CUNHA, M.F.; SGARBIERI, V.C.; DAMÁSIO, M.H. Effects of pretreatment with gamma rays or microwave on storage stability of dry beans. Journal of Agricultural and Food Chemistry, Easton, v. 41, n. 10, p. 1710 - 1715, 1993.

DALLA CORTE, A.; MODA-CIRINO, V.; SCHOLZ, M.B.S. Enviroment effect on grain quality in early common bean cultivars and lines. Crop Breeding and Applied Biotechnology, Londrina, v.3, n.3, p.193-202, 2003.

DOGBEVI, M.K.; VACHON, C.; LACROIX, M. Effect of gamma irradiation on the microbiological quality and on the functional properties of protein in dry red kidney beans (Phaseolus vulgaris) Radiation Physics and Chemistry, Oxford, v.57, p.265 - 268, 2000.

ESTEVES, A.M.; ABREU, C.M.P.; SANTOS, C.D. Comparação química e enzimática de seis linhagens de feijão (Phaseolus vulgaris L.). Ciência e Agrotecnologia, Lavras, v.26, n.5, p.999$1005,2002$.

GARCIA-VELA, L.A.; STANLEY, D.W. Water-holding capacity in hard-to-cook bean (P. vulgaris L.): effect of $\mathrm{pH}$ and ionic strength. Journal of Food Science, Chicago, v.54, n.4, p.10801081, 1989.

IYER, V.; SALUNKHE, D.K.; SATHE, S.K.; ROCKLAND, L.B. Quick-cooking beans (Phaseolus vulgaris L.): I. investigation on quality. Qualitas Plantarum Plant Foods for Human Nutrition, Netherlands, v. 30, p. 27-43, 1980.

JACKSON, G.M.; MARSTON, E.V. Hard-to-Cook phenomen in beans: Effects of accelerated Storage on Water Absorption and Cooking Time, Journal of Food Science, Chicago, v. 46, n. 3, p. $799-803,1981$.

LAM-SANCHEZ, A.; DURIGAN, J.F.; CAMPOS, S.L.; SILVESTRE, S.R.; PEDROSO, P.A.C.; BANZATTO, D.A. Efeitos da época de semeadura sobre a composição química e características físico-químicas de grãos de Phaseolus vulgaris L., Phaseolus angularis (Wild) Wright e Vigna unguiculata (L.) Walp. Alimentos e Nutrição, v.2, p.35-44, 1990.

LEMOS, L.B.; OLIVEIRA, R.S.; PALOMINO, E.C.;SILVA, T.R.B. Características agronômicas e tecnológicas de genótipos de feijão do grupo comercial Carioca. Pesquisa Agropecuária Brasileira, Brasília, v.39, n. 4, p.319-326, abr 2004.

MORRIS, H.J.; OLSON, R.L.; BEAN, R.C. Processing quality of varieties and strains of dry beans. Food Technology, Chicago v. 4 , p. 247-251, 1950.

NASCIMENTO, L.M. Efeito da radiação gama $\left({ }^{60} \mathrm{Co}\right)$ nas propriedades físico-químicas e sensoriais de feijões envelhecidos (Phaseolus vulgaris). 1992. $135 \mathrm{f}$. Tese (Doutorado em Ciência de Alimentos) - Faculdade de Ciências Farmacêuticas, Universidade São Paulo, São Paulo, 1992.

RAO, V.S.; VAKIL, U.K. Effects of gamma-radiation on cooking quality and sensory attributes for four legumes. Journal of Food Science, Chicago, v. 50, p. 372 - 379, 1989. 
RIOS, A.O.; ABREU, C.M.P.; CORREAA, A.D. Efeito da estocagem e das condições de colheita sobre algumas propriedades físicas, químicas e nutricionais de três cultivares de feijão (Phaseolus vulgaris, L.). Ciência e Tecnologia de Alimentos, Campinas, v.23, p. $39-45,2003$.

RODRIGUES, J.A.; RIBEIRO, N.D.; LONDERO, P.M.G. Correlação entre absorção de água e tempo de cozimento de cultivares de feijão. Ciência Rural, Santa Maria, v. 35, n.1, p. 209-214, 2005.

VIEIRA, C.R.; CABRAL, L.C.; PAULA, A.C.R. Caracterização física e tecnológica de seis cultivares de soja plantadas no Brasil. Ciência e Tecnologia de Alimentos, Campinas, v.17, n.3, p. 291 - 294, 1997.
VIEIRA, R.F.; ROMEIRO, E.M.C.; SOUZA, L.R.P.; DONZELLI, M.F.; VIEIRA, V. Tempo de cocção, rendimento alimentar e aceitabilidade de feijões secos dos gêneros Vigna e Phaseolus. Revista Ceres, Viçosa, v.36, p.525 - 537, 1989.

VILLAVICENCIO, A.L.C.H. Avaliação dos efeitos da radiação ionizante de ${ }^{60} \mathrm{Co}$ em propriedades físicas, químicas e nutricionais dos feijões Phaseolus vulgaris L. e Vigna unguiculata (L.) Walp. 1998. 138 f. Tese (Doutorado em Ciência dos Alimentos) - Faculdade de Ciências Farmacêuticas, Universidade de São Paulo, São Paulo, 1998.

WANG, H.L.; SWAIN, E.H.; HESSELTINE, C.W.; HEATH, H.D. Hydration of whole soybeans affects solids losses and cooking quality. Journal of Food Science, Chicago, v.44, n.5, p. 1510 1513, 1979. 